\title{
MR-arthrography and CT-arthrography in sports-related glenolabral injuries: a matched descriptive illustration
}

\author{
Mohamed Jarraya ${ }^{1,2,3}$ (D) Frank W. Roemer ${ }^{1,2,4}$ - Heather I. Gale ${ }^{5}$. \\ Philippe Landreau $^{1} \cdot$ Pieter D'Hooghe $^{1} \cdot$ Ali Guermazi ${ }^{1,2}$
}

Received: 28 September 2015 /Revised: 14 December 2015 / Accepted: 18 December 2015 / Published online: 8 January 2016

(C) The Author(s) 2016. This article is published with open access at Springerlink.com

\begin{abstract}
The combination of a large range of motion and insufficient bony stabilization makes the glenohumeral joint susceptible to injuries including dislocation in young athletes. Magnetic resonance arthrography (MR-arthrography) and computed tomography arthrography (CT-arthrography) play an important role in the preoperative workup of labroligametous injuries. This paper illustrates MR-arthrography and CT-arthrography findings acquired at the same time on the same subjects to illustrate common causes and sequelae of shoulder instability.

Teaching Points

- MR-arthrography and CT-arthrography are equivalent for SLAP and full-thickness rotator cuff tears.

- CT-arthrography is superior in evaluating osseous defects and cartilage surface lesions.

- MR-arthrography is superior in evaluating intrasubstance and extra-articular tendinous injuries.
\end{abstract}

Keywords Magnetic resonance imaging · Multislice computed tomography $\cdot$ Arthrography $\cdot$ Shoulder $\cdot$ Sports injuries

Mohamed Jarraya

mohamedjarraya@gmail.com

1 Aspetar Orthopaedic and Sports Medicine Hospital, Doha, Qatar

2 Department of Radiology, Boston University School of Medicine, 820 Harrison Avenue, FGH Building, 3rd floor, Boston, MA, USA

3 Department of Radiology, Mercy Catholic Medical Center, Darby, PA, USA

4 Department of Radiology, University of Erlangen-Nuremberg, Erlangen, Germany

5 Department of Radiology, Massachusetts General Hospital, Harvard Medical School, Boston, MA, USA

\section{Introduction}

The combination of a large range of motion and insufficient bony stabilization makes the glenohumeral joint susceptible to injuries, including dislocation and subsequent chronic instability especially in young active subjects [1]. Despite technological advancement of modern MRI systems, magnetic resonance arthrography (MR-arthrography) is considered the gold standard for assessment of instability and pre-operative workup for shoulder ligaments and labral injuries [2].

The shoulder joint is characterized by complex stabilizing mechanisms (static and dynamic), additional physiologic anatomic variants (e.g., sublabral foramen, Buford complex, and meniscoid labrum), and various pathologic presentations, which results in challenging imaging and even arthroscopic interpretation. Indeed, a surgical study showed relatively poor inter-rater reliability $(<40 \%)$ among orthopaedic surgeons for the exact description of anatomic structures such as the inferior glenohumeral ligament (IGHL) or the bony glenoid size during shoulder arthroscopy [3]. This fact highlights the important role of radiology in the assessment of shoulder instability using either MR-arthrography or computed tomography arthrography (CT-arthrography).

MR-arthrography and CT-arthrography yield comparable results for the detection and classification of sublabral recesses, SLAP, and full thickness rotator cuff tears [4, 5]. Although the degree of glenoid bone loss and the medial orientation of Hill Sachs lesions as detected on MR imaging has been shown to be significantly associated with engaging HillSachs on physical examination [6], a study directly comparing MR-arthrography and CT-arthrography in preoperative planning of anterior shoulder instability, showed limitations of MR-arthrography in detecting glenoid rim fractures and inferior glenohumeral ligaments injuries, such as humeral avulsion of gleno-humeral ligament (HAGL). This latter entity can 
Table 1 Summary of technical recommendations and correspondent indications

\begin{tabular}{ll}
\hline Technical recommendation & Indication \\
\hline $\begin{array}{l}\text { Injection approach } \\
\text { • Anterior }\end{array}$ & Posterior instability \\
• Posterior & Anterior instability \\
Preferred imaging modality & \\
- CT Arthrography & 1/ Cartilage surface abnormality \\
& 2/ Osseous defects \\
- MR Arthrography & 1/ Intra-substance tendinous injuries \\
& 2/ Extra-articular tendinous injuries \\
- MR or CT Arthrography & 1/ SLAP tear \\
& 2/ Full thickness rotator cuff tear \\
& 3/ Labro-ligamentous complex injuries \\
\hline
\end{tabular}

\section{a}
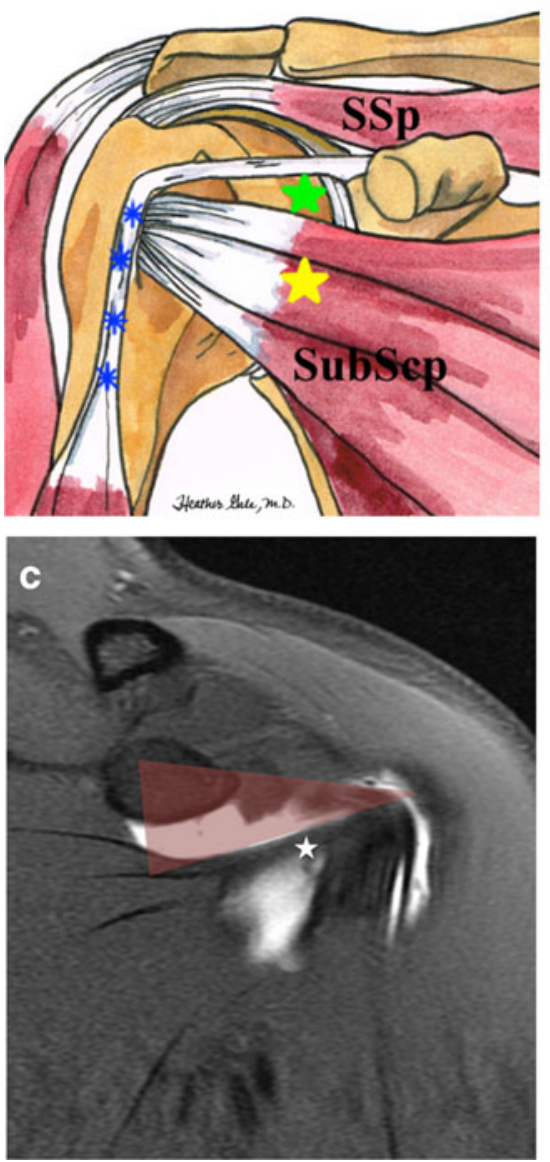

Fig. 1 a Diagram showing the left shoulder in external rotation. Using the anteroinferomedial approach, the target needle crosses the subscapularis (SubScp) muscle (yellow star) while the rotator cuff interval (green star) lies between supraspinatus (SSp) and subscapularis (SubScp) muscles. The long head of the biceps tendon (blue asterisks) courses in the bicipital groove and is displaced laterally away from target site for the needle. b Sagittal T1-weighted TSE fat-suppressed MR arthrography image showing rotator interval anatomy. Anatomic structures are overlaid by different transparent colors. Rotator interval (red) lies between subscapularis tendon anteriorly (green), and easily be missed during surgery depending on the surgical approach; therefore, its preoperative radiological recognition is crucial for appropriate surgical planning [7]. Also, the use of 3-D reconstruction in CT was shown to be of value for preoperative prediction of the engagement of Hill Sachs lesions, and therefore useful in surgical planning [8]. In addition to its excellent three-plane resolution, CT-arthrography is favoured by a shorter time of image acquisition yielding more comfort for the patient, and is less susceptible to motion artefacts. CTarthrography is regarded as the reference standard for the evaluation of cartilage surface lesions [2], and is superior to MRarthrography for preoperative planning of anterior instability [9]. On the other hand, MR-arthrography is more accurate for suspected partial thickness rotator cuff pathology [5].
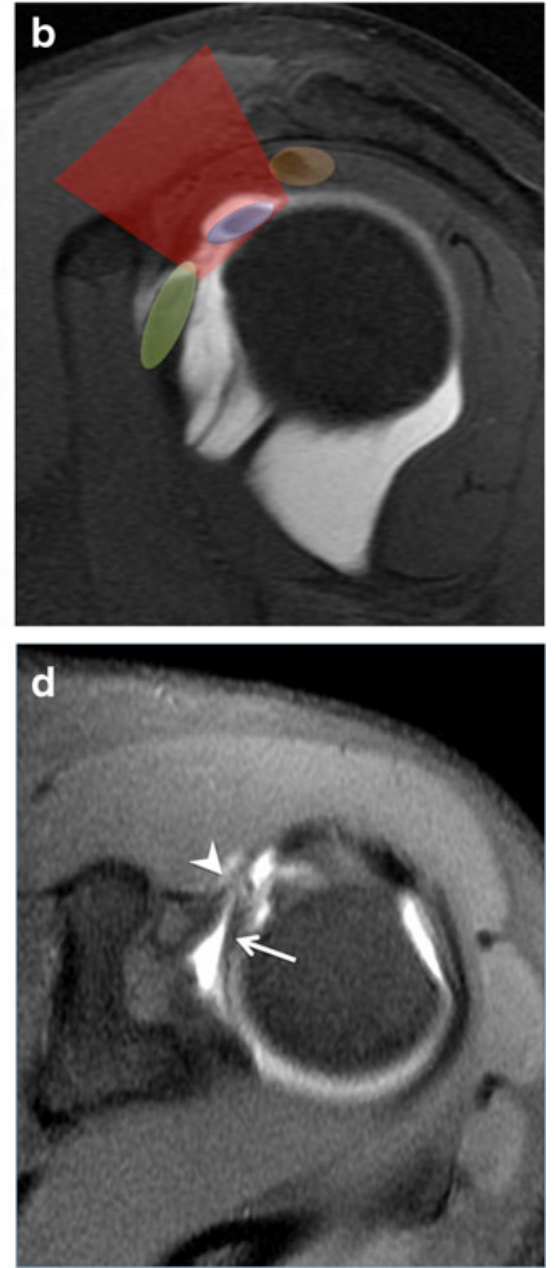

supraspinatus tendon posteriorly (orange). Long head of biceps brachii tendon (blue) traverses the rotator interval on its course from the bicipitallabral anchor to the bicipital groove. c Coronal T1-weighted TSE fatsuppressed MR arthrography image shows rotator interval (red transparent triangle), inferiorly limited by subscapularis tendon (asterisk), and superiorly by supraspinatus tendon (not shown). d Axial T1-weighted TSE fat-suppressed MR arthrography through rotator interval shows long head of biceps brachii tendon (arrow), and coracohumeral ligament (arrowhead) 

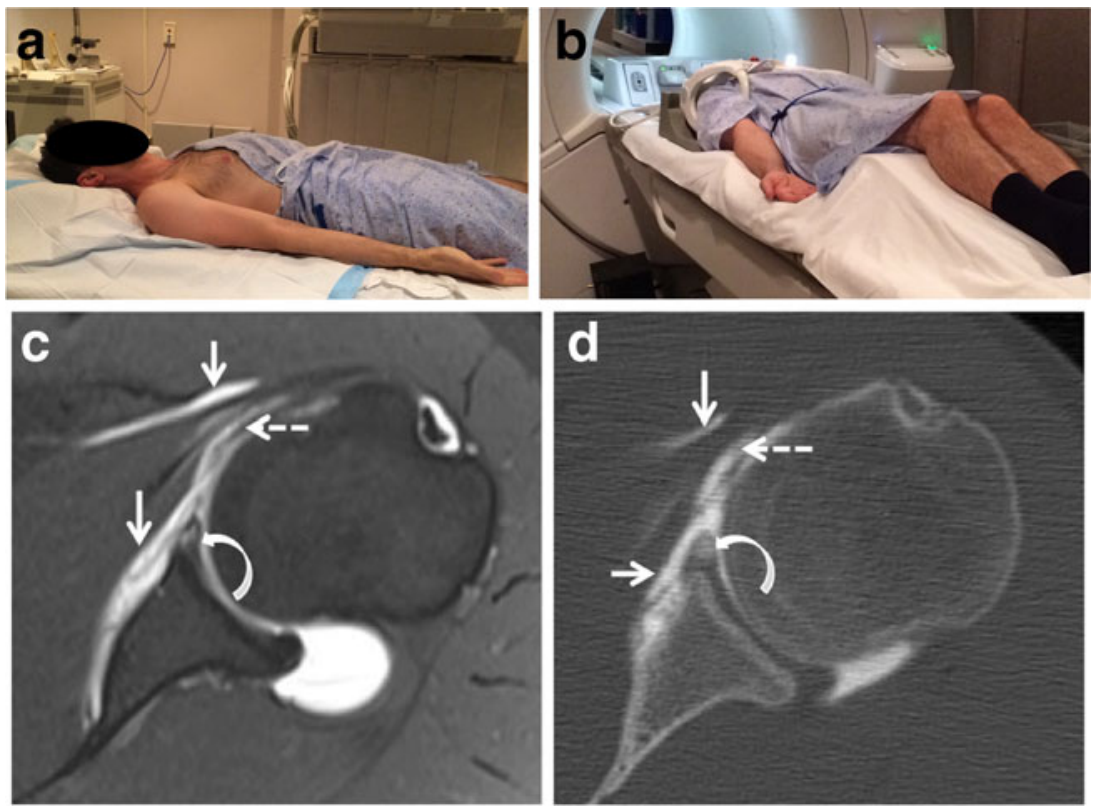

Fig. 2 a, b Patient positioning. Patient is placed supine, head first, arm along the body, and the arm in external rotation for both fluoroscopy guided injection (a), and subsequent MR examination (b). c, d Drawbacks of the anteroinferomedial approach in shoulder CT arthrography and MR arthrography. Axial (c) fat-suppressed T1- and (d) correspondent CT - arthrography image show distortion of the subscapularis muscle

While MR-arthrography is superior for the evaluation of intrasubstance ligamentous injuries and the extra-articular surface of the rotator cuff, CT-arthrography is better suited for the detection and evaluation of osseous defects (Table 1). The two modalities show comparable results otherwise, and the choice

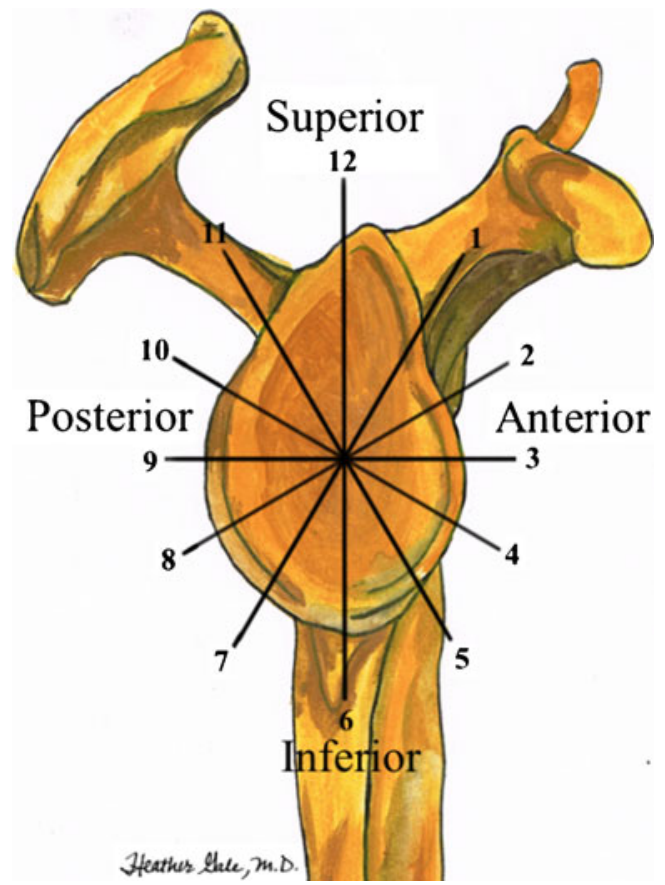

with peri- (straight arrows) and intra-muscular (dashed arrow) contrast media extravasation caused by the needle traversing the subscapularis muscle. In addition, it is unclear whether intralabral contrast is also related to needle misplacement or actual tear (curved arrow). This can add difficulty to the interpretation. An anterior-rotator interval approach would have been optimal in this case

is partly based on the specific indication for each patient as well as the reader's experience in each modality.

This pictorial review aims to present MR-arthrography and CT-arthrography findings acquired at the same time on the same subjects to illustrate common causes and sequelae of shoulder instability.

\section{Technique}

The shoulder joint may be approached anteriorly or posteriorly for injection. The posterior approach offers the benefit of minimizing anterior extravasation, which is particularly important when assessing suspected anterior instability. The posterior approach is reported to be more comfortable [10]; however, the anterior approach seems to be more popular with different portals described (Fig. 1). The junction of the middle and lower thirds of the humeral head is commonly targeted for arthrography injection. However this method can cause distortion of the inferior glenohumeral ligament and the anteroinferior labrum (Fig. 2). A modified anterior approach targeting the rotator cuff interval seems, therefore, to be more appropriate in suspected anterior instability [11] (Fig. 1). For intra-articular injection, both ultrasound and fluoroscopy guidance are comparable with regard to successful rate, pain score, and duration of injection, however ultrasound guidance injection has the intrinsic benefit of being non-ionizing [12].

Fig. 3 Diagram shows the clock-like division of the glenoid rim and it four sectors: inferior, posterior, anterior and superior 
Fig. 4 Normal variants around the superior labrum and the biceps anchor. a, b Axial (a) and sagittal (b) non fat-suppressed proton density-weighted images show a missing anterior superior labrum. The middle gleno-humeral ligament appears thick and of cord-like appearance

representative of a so-called Buford-complex (arrows). c Another example of a Buford complex on a fat-suppressed proton density-weighted image (arrows). d Coronal fat-suppressed proton-density-weighted image shows a meniscoid shaped labrum (arrow). e Axial T1-weighted MR arthrography image shows a socalled sublabral foramen (arrow). f, $\mathbf{g}$ The attachment of the biceps tendon at the glenoid may show different appearances. Proton density-weighted fat suppressed (f) and nonfat-suppressed (g) images of two different patients show a socalled Type II bicipito-labral complex representing a recess at the labral attachment (arrows). Such recesses point medially and as such may be distinguished from so-called SLAP II lesions that are commonly pointing towards the lateral aspect of the biceps anchor/ proximal tendon
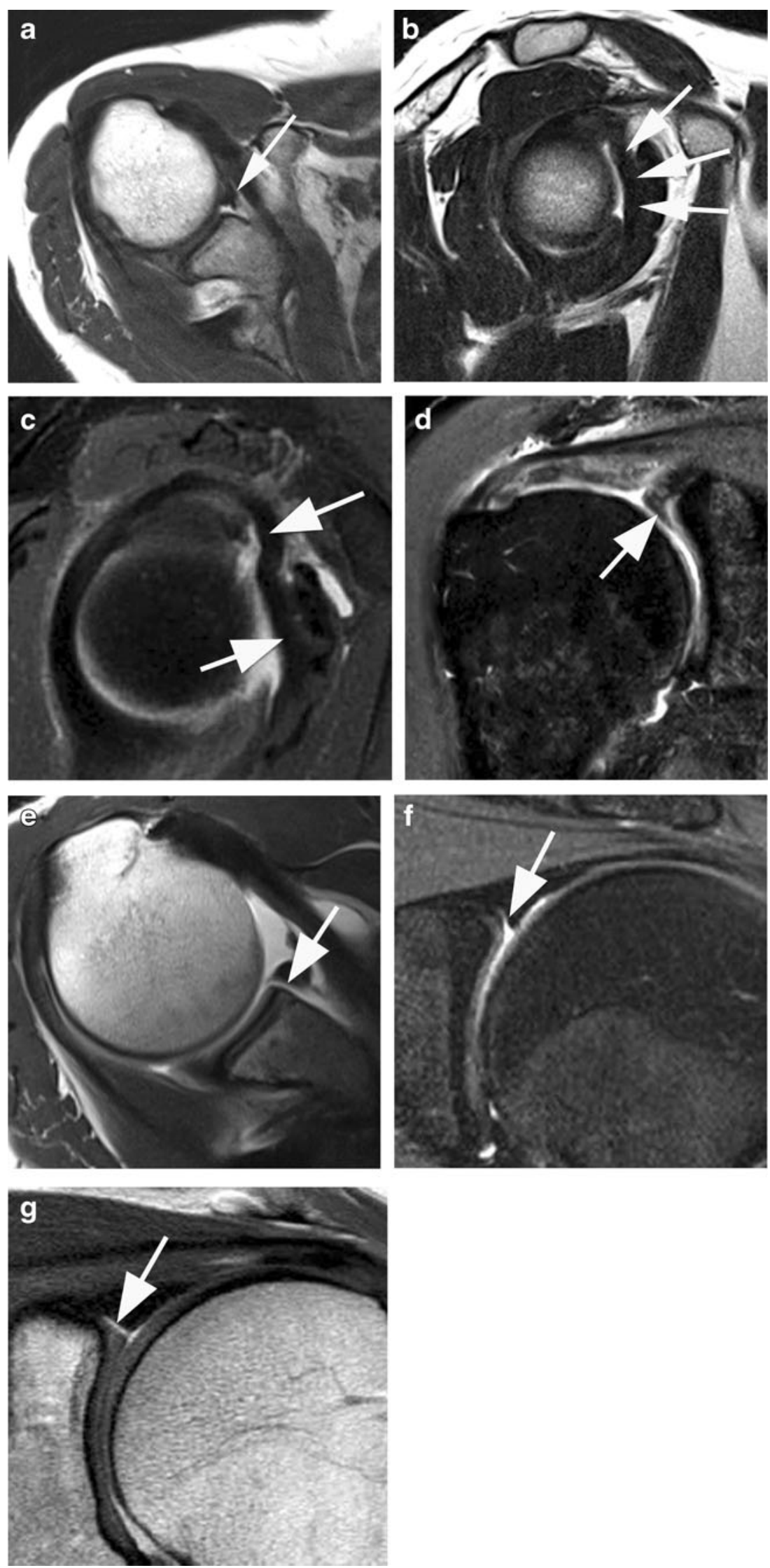


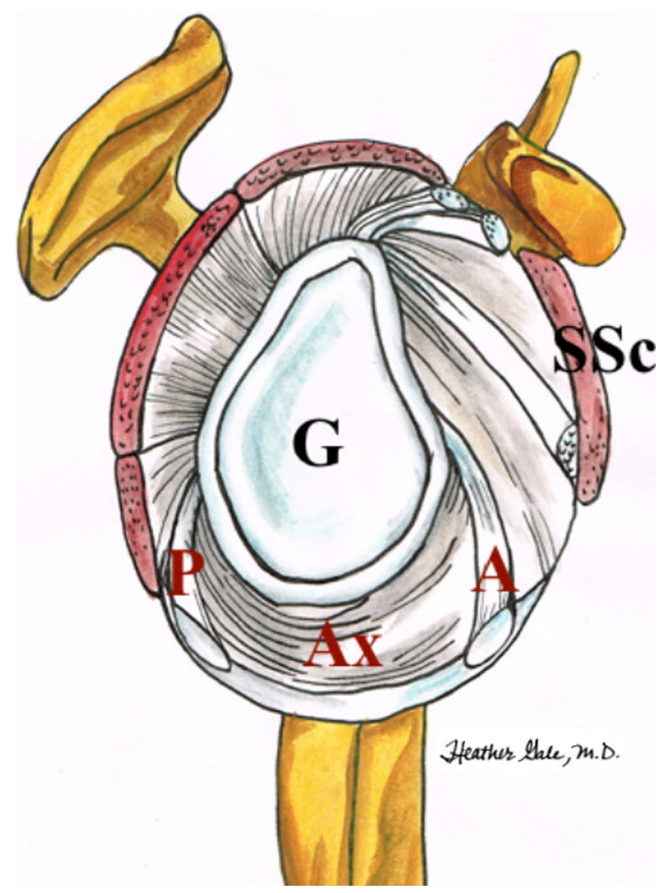

Fig. 5 Diagram shows the inferior glenohumeral ligament with its anterior $(A)$, posterior bands $(P)$ and axillary pouch $(A x)$. $G$ glenoid cavity, $S S c$ Subscapularis muscle

For our study, $0.1 \mathrm{~mL}$ of gadoterate meglumine (Dotarem ${ }^{\circledR}$ , Guerbet, Villepinte, France) mixed with $8 \mathrm{~mL}$ of saline, $7 \mathrm{~mL}$ iodinated contrast iohexol (OmnipaqueTM 300, GE Healthcare, Princeton, NJ, USA), and $5 \mathrm{~mL}$ of $1 \%$ lidocaine (final gadolinium dilution ratio of 1:200) was injected with a $22 \mathrm{G}$ needle into the glenohumeral joint with fluoroscopic guidance by using an anterior approach. After the injection, gentle passive mobilization of the shoulder was performed to allow diffusion of the contrast material in the joint cavity. For both imaging modalities, patient position was the same: supine, head first, arm along the body, and the arm in external rotation.

Spiral CT was performed immediately after joint opacification by using a 40-detector helical CT scanner (Sensation 40TM, Siemens Healthcare, Erlangen, Germany). After a frontal projection scout image, a 10-15-s scanning was performed to image the volume from the top of the acromioclavicular joint to the lower margin of the axillary recess of the glenohumeral joint. Acquisition parameters were $120 \mathrm{kV}, 315 \mathrm{mAs}, 12-\mathrm{mm}$ collimation beam, 25- cm field of view, effective pitch of 0.3 , effective thickness of about $0.8 \mathrm{~mm}$, and $512 \times 512$ matrix, bone convolution kernel (B75h); $2.0-\mathrm{mm}$ slice thickness, no gap. From images acquired in the axial plane, oblique and sagittal reformations were constructed to mirror the MRI planes, using the following parameters: slice thickness $=2.0 \mathrm{~mm}$, reconstruction increment $=2 \mathrm{~mm}$, Kernel $=$ B75 Sharp + , Window $=$ bone, pitch $=0.9$. CT dose length product ranged between 390 and $600 \mathrm{mGy} \mathrm{cm}$ depending on the size of the patient. X-Ray exposure (dose area product) ranged between $1.69 \mu \mathrm{Gy} \mathrm{m} 2$ and $54.48 \mu \mathrm{Gy} \mathrm{m} 2$.

Immediately after the CT examination, MR arthrography was performed with a $1.5 \mathrm{~T}$ large bore MR system (EspreeTM, Siemens Healthcare, Erlangen, Germany), using a dedicated 4channel shoulder array coil supplied by the manufacturer. MR sequences included axial and coronal T1-weighted turbo spin echo (TR: $480 \mathrm{~ms}$, TE: $13 \mathrm{~ms}$, No. Excitations: 2, Field of View: $18 \times 18 \mathrm{~cm}$ [axial] / $16 \times 16 \mathrm{~cm}$ [coronal], imaging matrix: $256 \times 192$, slice thickness: $3 \mathrm{~mm}$, interslice gap: $0.9 \mathrm{~mm}$ ).

\section{Shoulder anatomy}

Compared to other joints, the bony stabilization of the glenohumeral joint is insufficient. The glenoid cavity
Fig. 6 Inferior glenohumeral ligament. Sagittal (a) fatsuppressed T1-weighted MR-arthrography and (b) corresponding CT - arthrography images show the anterior band (curved arrow) and posterior band (straight arrow) of the inferior glenohumeral ligament
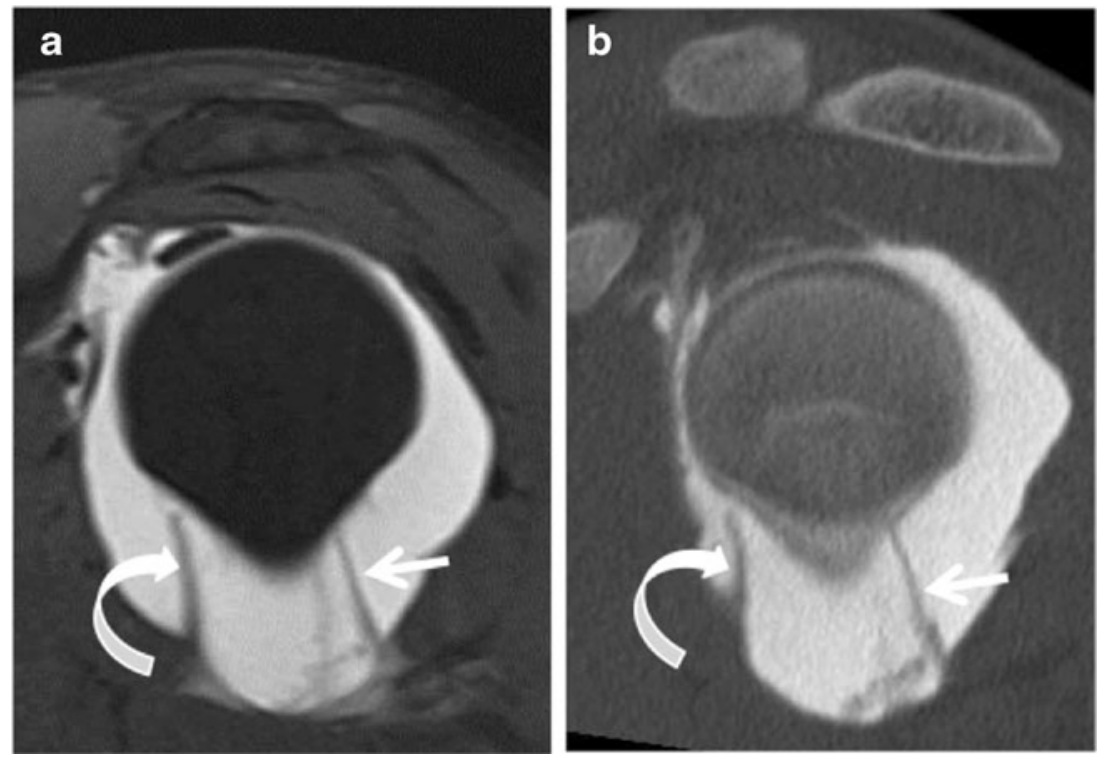
Fig. 7 Mechanisms of injury of SLAP lesions: a Acute from fall on outstretched arm (soccer goalkeeper, rugby player, etc.), or b chronic from superior traction to the biceps tendon from overhead sports (baseball pitcher)

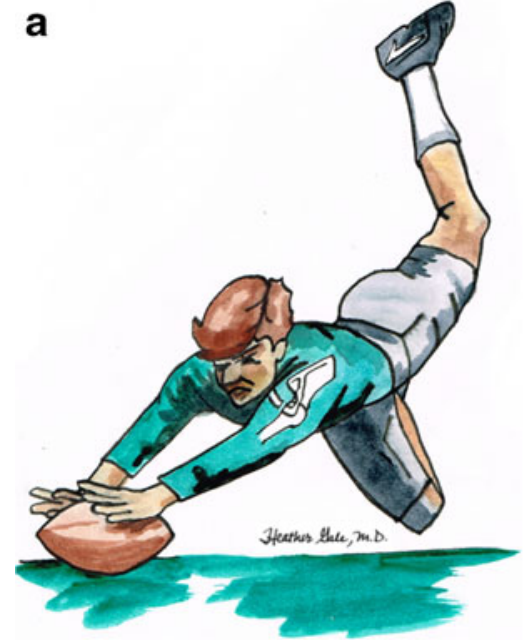

b

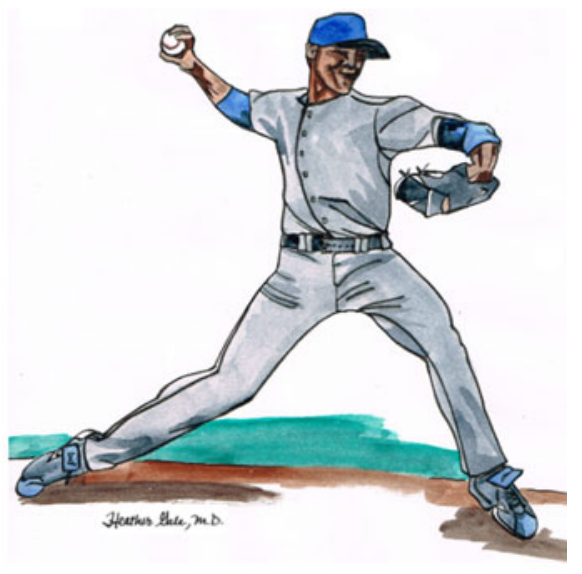

has a teardrop (pear), or oval shape. The latter is covered by the glenoid labrum in a circular fashion forming a functional unit with the capsule, the glenohumeral ligaments, long head of the biceps, and long head of the triceps. For the purpose of localizing abnormalities, the labrum is divided in clock-wise fashion (Fig. 3). The labrum is likened to the face of a clock, with the superior portion positioned at
12 o'clock and the inferior portion at 6 o'clock. By convention, the anterior portion is positioned at $3 \mathrm{o}$ 'clock and the posterior portion at 9 o'clock for both shoulders [13].

At its anterosuperior portion, the glenoid labrum anchors the to biceps tendon, called the labor-bicipital complex. Normal variants include the sublabral recess and foramen [13]. The Buford complex is rare and
Fig. 8 Drawing shows four main types of SLAP injury. a Type I. Fraying of the free edge of the superior labrum, intact biceps tendon (degenerative) (11-1 o'clock). b Type II. Most common type. Avulsion of superior labrum and biceps anchor (11-1o'clock). c Type III. Bucket-handle tear of the superior labrum with intact biceps anchor (11-1 o'clock). d Type IV. Extension of a bucket-handle of the superior labrum into biceps anchor (11-1 o'clock)
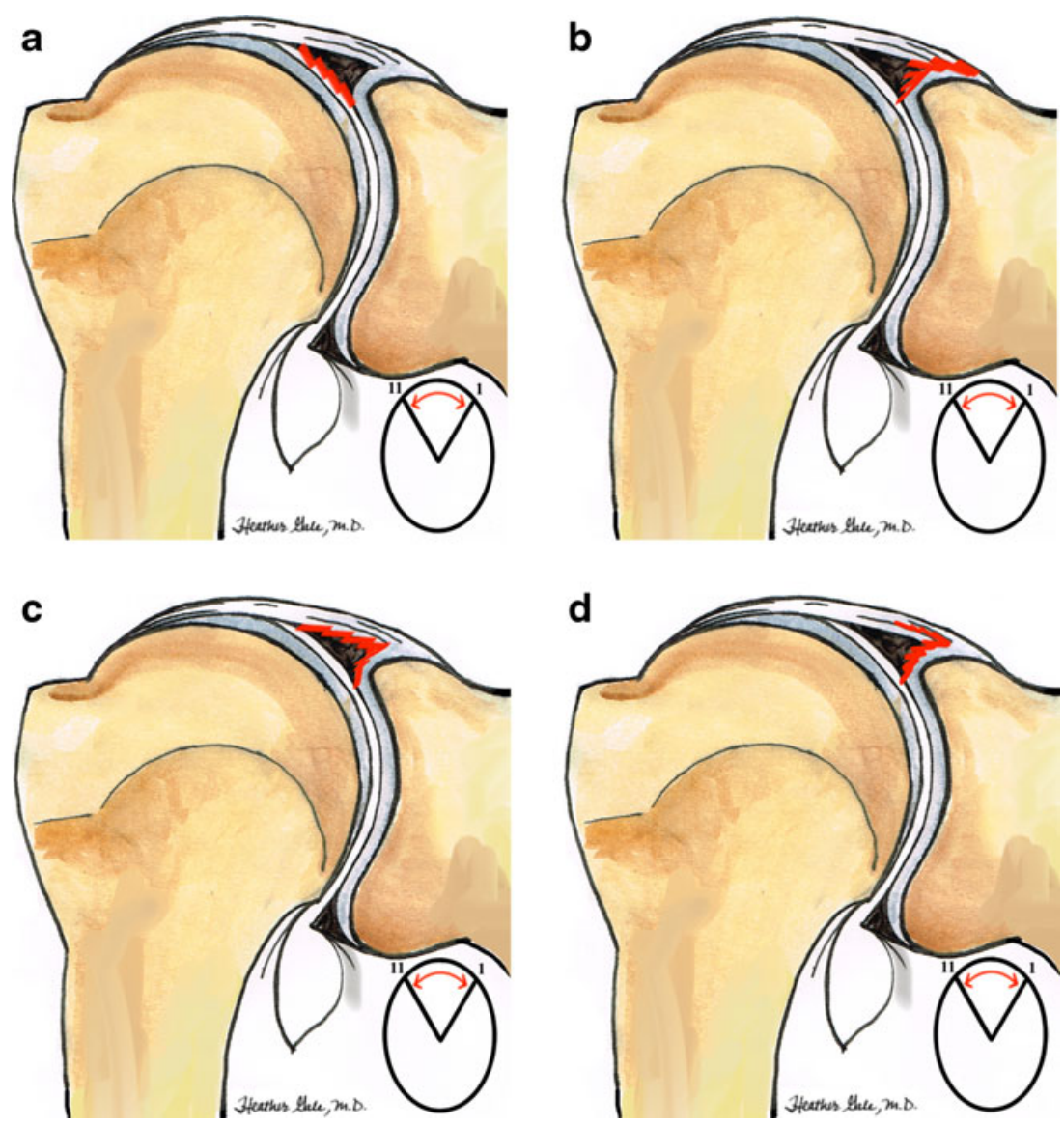
Fig. 9 Bucket-handle SLAP tear (Type 3) with Perthes lesion in a 23-year-old male athlete with a one-month history of right shoulder pain after a fall onto outstretched arm. a Coronal and c axial fat-suppressed T1-weighted MR arthrography with correspondent $\mathbf{b}$ coronal and $\mathbf{d}$ axial CT arthrography images. Presence of a Bucket handle tear of the labro-bicipital complex extending across the bicipital attachment (white plain arrows) and to the anteroinferior labrum (11-6 o'clock). The anteroinferior labrum is avulsed (black curved arrow) with medial stripping of an intact scapular periosteum (white arrowhead) consistent with a Perthes lesion. There is a cartilage defect of the anteroinferior labrum (dashed arrow). These findings can be classified either as a SLAP III (with regard to the bucket handle tear) with Perthes lesion, or SLAP $\mathrm{V}$ (with regard to the anteroinferior extension of the labral tear)
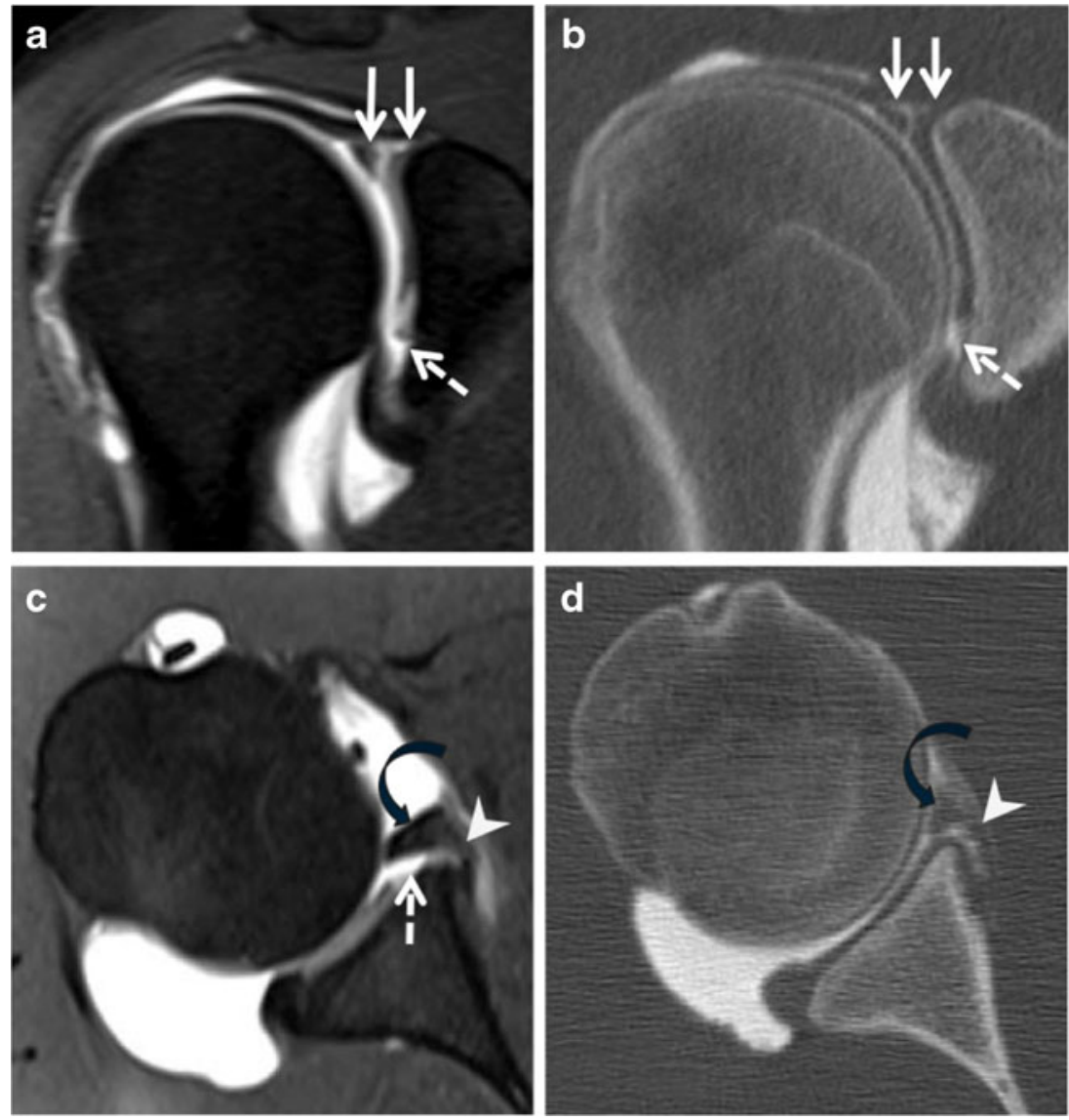

consists in the absent anterosuperior labrum replaced by a cordlike middle glenohumeral ligament (MGHL). The inferior part of the labrum anchors to the IGHL. Normal variants are optimally seen on axial and coronal images of CT and MR-arthrography [14] (Fig. 4).

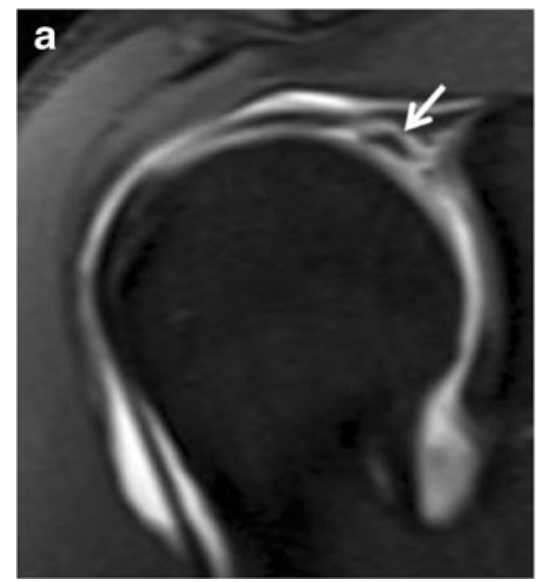

Fig. 10 SLAP tear type IV in a 20-year-old goalkeeper after a fall onto fully abducted right shoulder with immediate pain. Coronal (a) fatsuppressed T1-weighted MR arthrography and (b) corresponding coronal CT arthrography image. Bucket handle tear of the labrobicipital complex extending across the bicipital attachment (straight
The capsule consists of a complex system of circularly and radially arranged collagen fibres and is strengthened by multiple reinforcements, including the superior glenohumeral complex (SGHL), the MGHL, and the IGHL. The rotator interval is defined as the

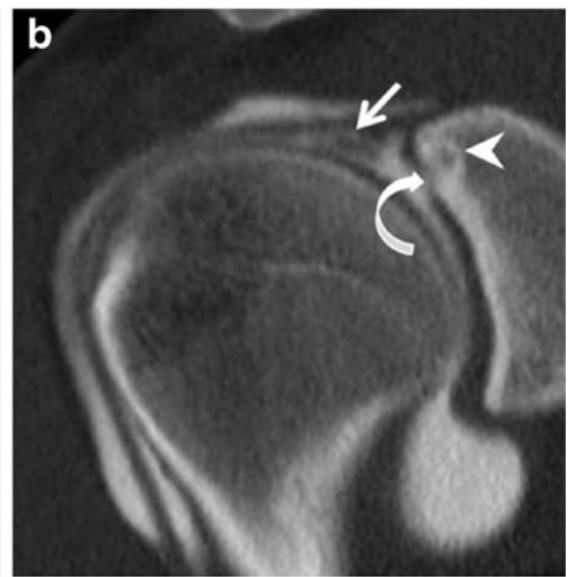

arrow). Note the presence of a small focal cartilage defect of the superior labrum only detected on the CT arthrography image (curved arrow) with adjacent subchondral cyst (arrowhead) suggesting a chronic chondropathy 
Fig. 11 Diagram showing extended SLAP tear classification according to direction of extension of labral tear $[13,19$, 20]

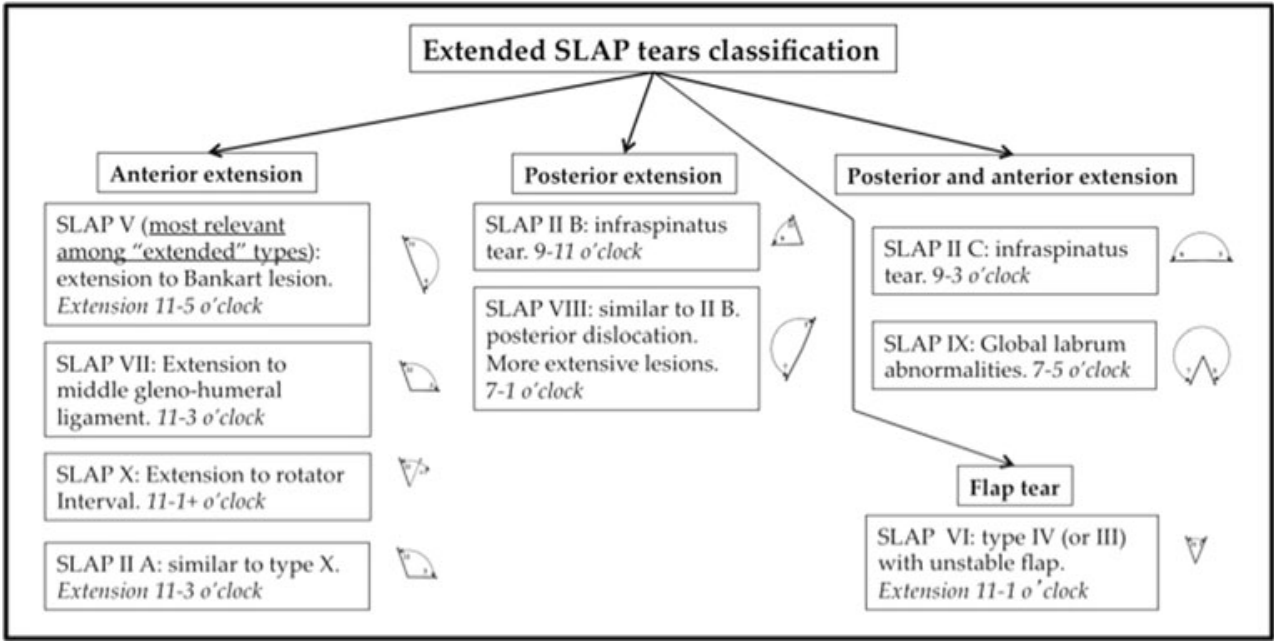

space between the supraspinatus and subscapularis muscles.

The MGHL is variably developed and radiates from the superior glenoid tubercle to the inferior parts of the lesser tuberosity [15]. The IGHL consists of a strong anterior band and posterior band with the axillary recess residing between them, and is also called a hammock (Figs. 5, and 6). Both parts of the ligament stabilize the humeral head in the vulnerable abduction and external rotation movements [15].

\section{Superior labral anteroposterior (SLAP) tears}

SLAP lesions are defined as a pathologic labral separation from the biceps anchor, with variable extension to either the
Fig. 12 SLAP tear type V with rotator cuff lesion in a 38-year-old male athlete with right shoulder pain. a Coronal and $\mathbf{c}$ axial fatsuppressed T1-weighted MR arthrography and correspondent $\mathbf{b}$ coronal and $\mathbf{d}$ axial CT arthrography images show an intermediate undersurface tear of the anterior portion of the supraspinatus (dashed arrows). Note also the presence of a labrobicipital tear extending across the biceps attachment (curved arrow) and inferiorly (11-6 o'clock). Hypertrophy (empty arrow) and linear tear (curved arrow) of the inferior labrum communicating with a small paralabral cyst (arrowhead) are noted. This constellation of findings is in keeping with SLAP V lesion
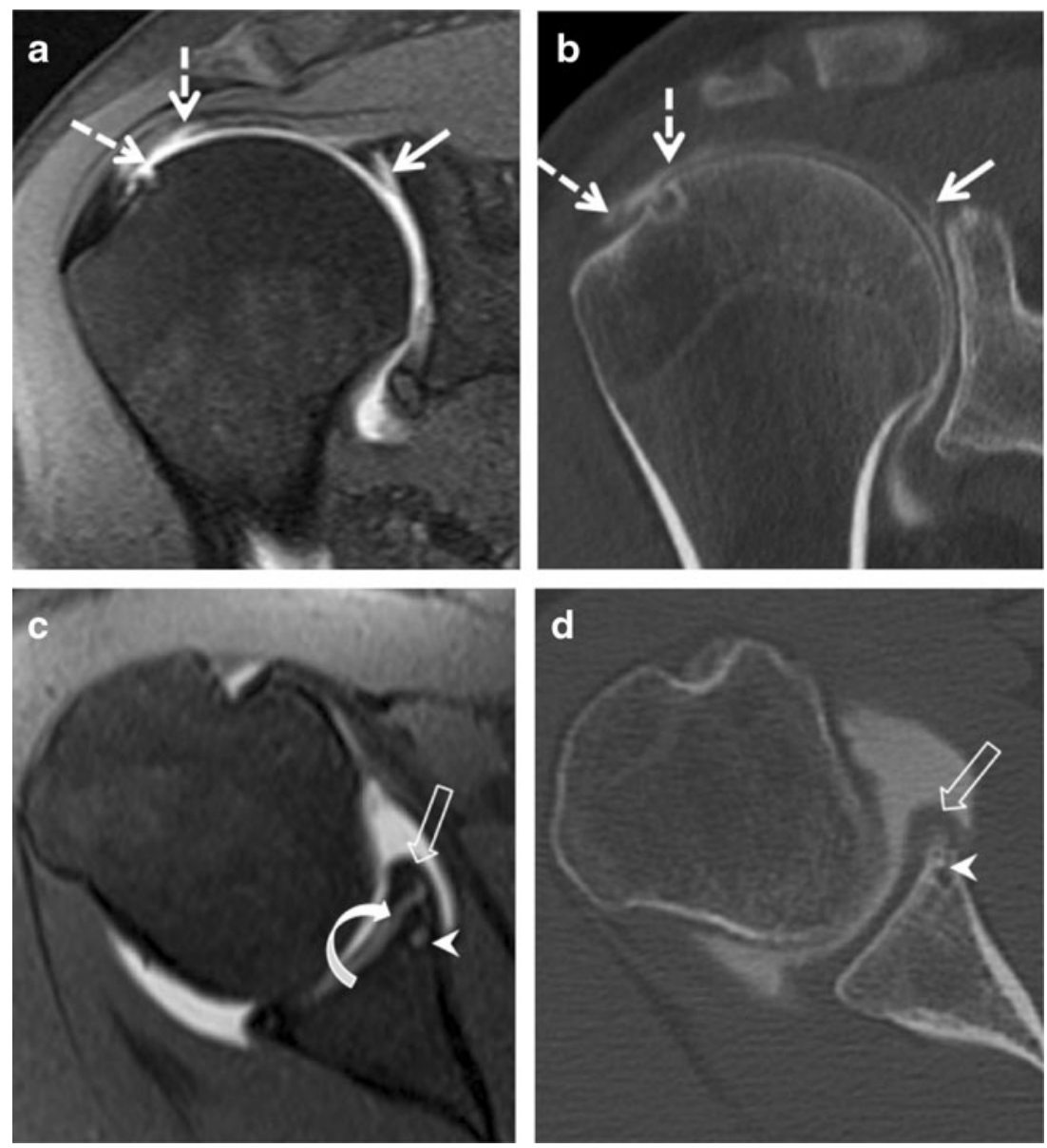


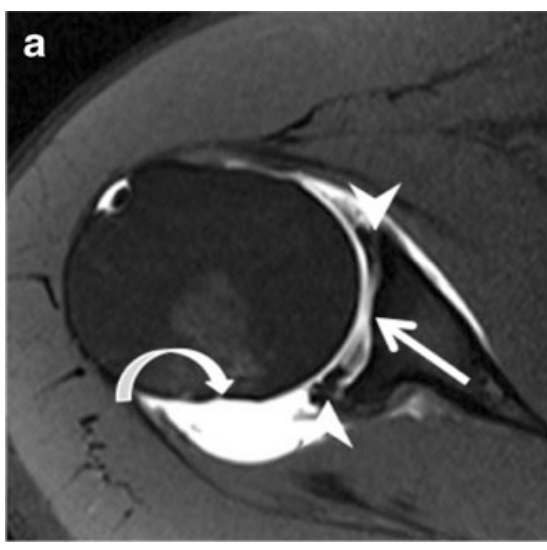

Fig. 13 Global labral (SLAP IX) tear in a 24-year-old male athlete with a 10-month history of right shoulder pain. Axial (a) fat suppressed T1weighted MR arthrography and (b) corresponding axial CT arthrography images. Cartilage defect (straight arrow) is more conspicuous on $\mathrm{CT}$ arthrography than on the MR arthrography image.

anterior or posterior portion of the labrum. Determining the type of attachment of the biceps to the superior labrum and adjacent supraglenoid notch, as well as the presence of anatomic variations, is the first step in accurate evaluation of this region. SLAP tears are commonly found in throwing athletes because of the high stresses of repetitive overhead throwing, which results in excessive traction to the biceps tendon. Snyder found that the mechanism of injury most commonly was related to an acute trauma: fall on an outstretched arm with the shoulder positioned in abduction and slight forward flexion at the time of impact [16]. Andrews et al. reported high prevalence of SLAP lesions among overhead throwing athletes (baseball pitchers) suggesting a chronic injury [17] (Fig. 7).

SLAP tears were initially classified into four types (Fig. 8) [16]: Type I: fraying of the free edge of the superior labrum with a normal biceps anchor is thought to be a degenerative process and uncommon source of pain [18]. Type II: fraying

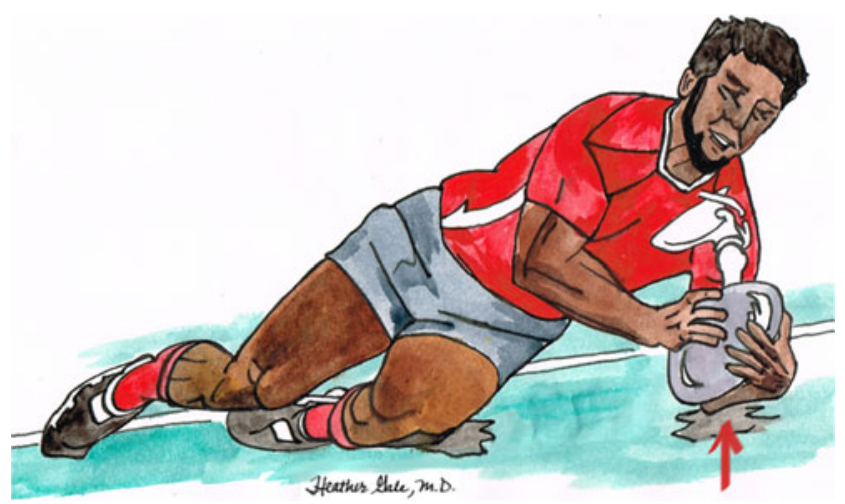

Fig. 14 Drawing shows the mechanism of posterior shoulder injury in rugby player falling on a flexed elbow and shoulder (forced adduction and internal rotation)

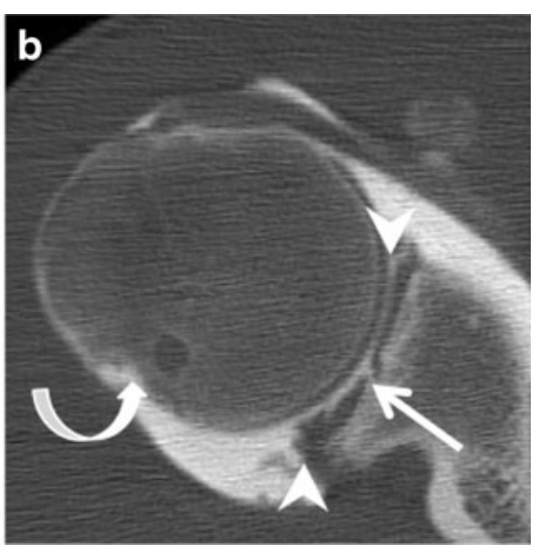

Circumferential labral abnormalities (arrowheads) extending across the labro-bicipital complex (not shown) in keeping with SLAP tear type IX. A subtle osseous impaction of the posterosuperior aspect of the humeral head (Hill-Sachs defect) (curved arrow) is detected suggesting prior anterior dislocations

of the free edge of the superior labrum associated with a detached biceps anchor from the superior glenoid tubercule, is the most commonly encountered during arthroscopy. Type III: bucket handle tear of a meniscoid superior labrum with an otherwise normal biceps tendon attachment (Fig. 9). Type IV: bucket handle tear of a meniscoid superior labrum with extension of the tear into the biceps tendon (Fig. 10). Other later types have been introduced and are summarized in Fig. $11[13,19,20]$. For instance, SLAP Type V (Fig. 12) is defined by associating a SLAP tear with a Bankart type labral lesion, while type IX is defined by a global labral injury (Fig. 13).

\section{Anterior-inferior labro-ligamnetous injuries}

The most common labral lesion is the classic labral Bankart lesion, in which the anterior-inferior labrum is separated from the glenoid. The anterior labroligamentous periosteal sleeve avulsion (ALPSA) occurs after multiple dislocations and consists in anteroinferior labral avulsion leading to medial displacement and inferior rotation of the labroligamentous complex with intact periosteum, in a sleeve like fashion [21].

The Perthes lesion is a variation of the Bankart lesions and occurs when the scapular periosteum remains intact but is stripped medially and the anterior labrum is avulsed from the glenoid but remains partially attached to the scapula by the intact periosteum [21] (Fig. 9).

The glenolabral articular disruption (GLAD) is a superficial tear of the anteroinferior labrum associated with an articular cartilage lesion of the anterior inferior quadrant of the glenoid. The injury is thought to result from glenohumeral impaction while the arm is abducted and externally rotated [21]. 


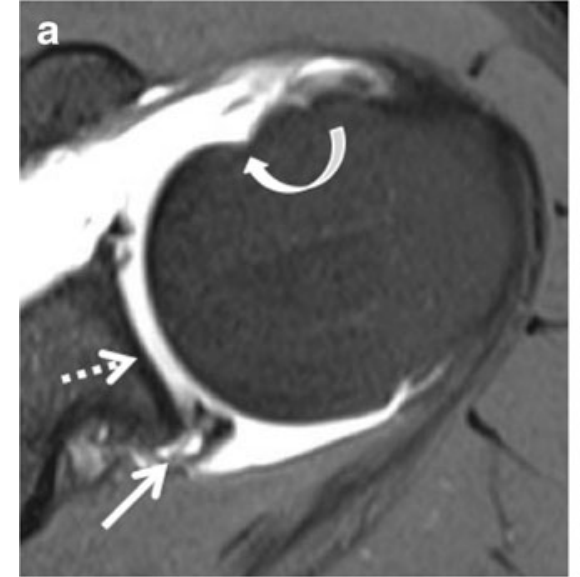

Fig. 15 Posterior instability in a 32-year-old male athlete following a ski fall on a flexed elbow and shoulder a year earlier. Axial (a) fat-suppressed T1-weighted MR arthrography and (b) corresponding CT arthrography images. Posterior labral tear consistent with reverse Bankart tear (straight

The HAGL lesion consists in an isolated tear of the IGHL at its humeral insertion following vigorous shoulder dislocation. HAGL may be caused by traumatic hyperabduction with external rotation of the arm. Different activities may be involved, such as rugby, snow or water skiing, surfing, football, volleyball, basketball, ice hockey, wrestling, and boxing [22]. This type of injury represents a pitfall at both arthroscopy and open shoulder surgery, since it can easily be overlooked if the humeral neck is not specifically searched for this finding. It is therefore paramount that radiologists be aware of this entity for an accurate preoperative diagnosis. Contrast extravasation can be observed at the insertion site [21].

The Hill-Sachs lesion is a posttraumatic impaction injury along the posterolateral aspect of the humeral head, resulting from repetitive anterior glenohumeral subluxation (Fig. 13).

\section{posterior glenuhumeral injuries}

Posterior labral tears typically result from a single event of axial loading on the arm while adducted, flexed, and internally rotated (Fig. 14), or from repetitive trauma, such as straight arm pass blocking in football, or bench pressing [23-25]. The patterns of injury are usually the reverse of those found following anterior dislocation. Tears that occur in the posterior labrum are referred to as reverse Bankart, whereas impaction of the anterosuperior humeral head gives rise to the reverse Hill Sachs defect (Fig. 15).

Kim classified labral injuries associated with posterior instability into three categories: separation without displacement, incomplete avulsion (concealed avulsion arthroscopy), and loss of contour, either from a flap tear or a chondrolabral erosion [26].

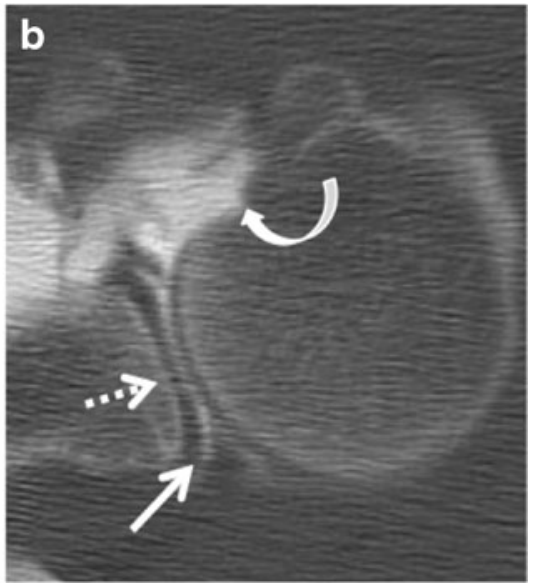

arrow). Cartilage thinning of the glenoid (dashed arrow) and anterosuperior humeral head impaction (curved arrow), consistent with reversed Hill-Sachs. The labral injury extends to the superior labrum (not shown)

\section{Conclusion}

Shoulder CT-arthrography and MR-arthrography yield very similar results in many clinical scenarios. Definition of the respective indications of both techniques depends on many factors, but it is primarily the relative availability and local experience. MR-arthrography is superior for visualization of the intra-articular portion of ligaments and depiction of bone marrow changes, while CTarthrography is the method of choice for assessment of articular cartilage surface lesions, fractures, and bony avulsions like osseous Bankart lesions.

\section{Compliance with ethical standards}

Conflict of interest - Mohamed Jarraya, Pieter D'Hooghe, and Heather I Gale have no conflict of interest to disclose.

- Frank W Roemer has shares in Boston Imaging Core Lab, LLC,

- Philippe Landreau has shares in Implanet, receives patent royalties from Medacta, and receives honoraria from Smith and Nephew.

- Ali Guermazi serves as a consultant for Genzyme, TissueGene, OrthoTrophix, AstraZeneca and Merck Serono. He has shares in Boston Imaging Core Lab, LLC.

Open Access This article is distributed under the terms of the Creative Commons Attribution 4.0 International License (http:// creativecommons.org/licenses/by/4.0/), which permits unrestricted use, distribution, and reproduction in any medium, provided you give appropriate credit to the original author(s) and the source, provide a link to the Creative Commons license, and indicate if changes were made.

\section{References}

1. Owens BD, Duffey ML, Nelson BJ, DeBerardino TM, Taylor DC, Mountcastle SB (2007) The incidence and characteristics of shoulder instability at the United States Military Academy. Am J Sports Med 35:1168-1173 
2. Lecouvet FE, Simoni P, Koutaissoff S, Vande Berg BC, Malghem J, Dubuc JE (2008) Multidetector spiral CT arthrography of the shoulder. Clinical applications and limits, with MR arthrography and arthroscopic correlations. Eur J Radiol 68:120-136

3. Sasyniuk TM, Mohtadi NG, Hollinshead RM, Russell ML, Fick GH (2007) The inter-rater reliability of shoulder arthroscopy. Arthroscopy 23:971-977

4. Waldt S, Metz S, Burkart A et al (2006) Variants of the superior labrum and labro-bicipital complex: a comparative study of shoulder specimens using MR arthrography, multi-slice CT arthrography and anatomical dissection. Eur Radiol 16:451-458

5. Oh JH, Kim JY, Choi JA, Kim WS (2010) Effectiveness of multidetector computed tomography arthrography for the diagnosis of shoulder pathology: comparison with magnetic resonance imaging with arthroscopic correlation. J Shoulder Elb Surg 19:14-20

6. Gyftopoulos S, Yemin A, Beltran L, Babb J, Bencardino J (2013) Engaging Hill-Sachs lesion: is there an association between this lesion and findings on MRI? AJR Am J Roentgenol 201:W633W638

7. Bui-Mansfield LT, Taylor DC, Uhorchak JM, Tenuta JJ (2002) Humeral avulsions of the glenohumeral ligament: imaging features and a review of the literature. AJR Am J Roentgenol 179:649-655

8. Cho SH, Cho NS, Rhee YG (2011) Preoperative analysis of the Hill-Sachs lesion in anterior shoulder instability: how to predict engagement of the lesion. Am J Sports Med 39:2389-2395

9. Acid S, Le Corroller T, Aswad R, Pauly V, Champsaur P (2012) Preoperative imaging of anterior shoulder instability: diagnostic effectiveness of MDCT arthrography and comparison with MR arthrography and arthroscopy. AJR Am J Roentgenol 198:661-667

10. Perdikakis E, Drakonaki E, Maris T, Karantanas A (2013) MR arthrography of the shoulder: tolerance evaluation of four different injection techniques. Skelet Radiol 42:99-105

11. Depelteau H, Bureau NJ, Cardinal E, Aubin B, Brassard P (2004) Arthrography of the shoulder: a simple fluoroscopically guided approach for targeting the rotator cuff interval. AJR Am J Roentgenol 182:329-332

12. Ng AW, Hung EH, Griffith JF, Tong CS, Cho CC (2013) Comparison of ultrasound versus fluoroscopic guided rotator cuff interval approach for MR arthrography. Clin Imaging 37:548-553
13. Mohana-Borges AV, Chung CB, Resnick D (2003) Superior labral anteroposterior tear: classification and diagnosis on MRI and MR arthrography. AJR Am J Roentgenol 181:1449-1462

14. De Maeseneer M, Van Roy F, Lenchik L et al (2000) CT and MR arthrography of the normal and pathologic anterosuperior labrum and labral-bicipital complex. Radiographics 20 Spec No:S67-81

15. Tischer T, Vogt S, Kreuz PC, Imhoff AB (2011) Arthroscopic anatomy, variants, and pathologic findings in shoulder instability. Arthroscopy 27:1434-1443

16. Snyder SJ, Karzel RP, Del Pizzo W, Ferkel RD, Friedman MJ (1990) SLAP lesions of the shoulder. Arthroscopy 6:274-279

17. Andrews JR, Carson WG Jr, McLeod WD (1985) Glenoid labrum tears related to the long head of the biceps. Am J Sports Med 13: 337-341

18. Nam EK, Snyder SJ (2003) The diagnosis and treatment of superior labrum, anterior and posterior (SLAP) lesions. Am J Sports Med 31:798-810

19. Maffet MW, Gartsman GM, Moseley B (1995) Superior labrumbiceps tendon complex lesions of the shoulder. Am J Sports Med 23:93-98

20. Morgan CD, Burkhart SS, Palmeri M, Gillespie M (1998) Type II SLAP lesions: three subtypes and their relationships to superior instability and rotator cuff tears. Arthroscopy 14:553-565

21. Woertler K, Waldt S (2006) MR imaging in sports-related glenohumeral instability. Eur Radiol 16:2622-2636

22. Carlson CL (2004) The "J" sign. Radiology 232:725-726

23. Sheehan SE, Gaviola G, Gordon R, Sacks A, Shi LL, Smith SE (2013) Traumatic shoulder injuries: a force mechanism analysisglenohumeral dislocation and instability. AJR Am J Roentgenol 201:378-393

24. de Beer J, Bhatia DN (2009) Shoulder injuries in rugby players. Int J Shoulder Surg 3:1-3

25. Tannenbaum E, Sekiya JK (2011) Evaluation and management of posterior shoulder instability. Sports Health 3:253-263

26. Kim SH, Kim HK, Sun JI, Park JS, Oh I (2004) Arthroscopic capsulolabroplasty for posteroinferior multidirectional instability of the shoulder. Am J Sports Med 32:594-607 